\title{
The Molecular Weight Distribution and Conformation of Citrus Pectins in Solution Studied by Hydrodynamics
}

\author{
Stephen E. Harding, ${ }^{a *}$ Gisela Berth ${ }^{b}$ Abigail Ball, ${ }^{a}$ \\ John R. Mitchell ${ }^{a} \&$ Jose Garcia de la Torre ${ }^{c}$
}

\author{
${ }^{a}$ Department of Applied Biochemistry \& Food Science, \\ University of Nottingham, Sutton Bonington LE12 5RD, UK \\ ${ }^{b}$ Akademie der Wissenschaften der DDR, Zentralinstitut für Ernährung, \\ Potsdam-Rehbrücke, FRG \\ 'Departamento de Quimica Fisica, Facultad de Ciencas, Universidad de Murcia, \\ 30100 Murcia, Spain
}

(Received 24 May 1990; accepted 4 July 1990)

\begin{abstract}
The molecular weight distribution of a citrus pectin has been analysed by a combined approach using gel-permeation chromatography with lowspeed sedimentation equilibrium. (I) A pectin preparation from citrus fruit was fractionated on Sepharose CL-2B/Sepharose CL-4B. (2) Weight average molecular weights of the fractions were determined by low speed sedimentation equilibrium in multichannel cells. (3) An absolute calibration for the column for this material was thereby defined. (4) The (lognormal) molecular weight distribution thus obtained is consistent with a weight average of $(90000 \pm 10000) \mathrm{g} / \mathrm{mol}$, obtained separately on unfractionated material, and consistent with a distribution obtained on the same material but using light scattering as the molecular weight probe.

The conformation of the pectin fractions in solution was studied in terms of: (1) the Wales-Van Holde parameter, $\left.k_{\mathrm{s}} / / \eta\right]$; (2) MarkHouwink-Kuhn-Sakurada plots of sedimentation coefficient and intrinsic viscosity data versus molecular weight; (3) rod models and (4) wormlike-coil models. The sedimentation data is consistent with a rod model (or a worm-like-coil with a large persistence length) with mass per unit length $\sim 430 \mathrm{~g} \mathrm{~mol}^{-1} \mathrm{~nm}^{-1}$. The intrinsic viscosity data is also consistent with a rod model but shows some anomalous features which may be suggestive of worm-like-coil behaviour at higher molecular weight, although it is not possible to fit this data with a realistic value of the mass per unit length.
\end{abstract}

*To whom correspondence should be addressed.

Carbohydrate Polymers 0144-8617/91/\$03.50 - (c) 1991 Elsevier Science Publishers Ltd, England. Printed in Great Britain 


\section{INTRODUCTION}

Pectins are a class of polyuronides used in a variety of commercial applications. For example, in the food industry the traditional use of high methoxyl pectins has been as a gelling agent in jams and preserves. In recent years pectin has found increasing use as a stabiliser in drinking yoghurt products: the addition of pectin preventing separation of the product after pasteurisation and enabling aseptically filled drinking yoghurts to be used (May, 1990). The performance of pectins in such applications depends critically on their average molecular weight and distribution of molecular weights (Deckers et al., 1986). For example, preparations with molecular weight distributions possessing broad lowmolecular-weight tails are known to yield poor gelling properties (Kim et al., 1978).

In an earlier paper (Berth et al., 1990) the molecular weight distribution of a citrus pectin determined by gel-permeation chromatography (GPC) on a Sepharose CL-2B/Sepharose CL-4B column - calibrated using osmotic pressure or light-scattering measurements - was reported. Agreement between the two procedures could be obtained only if correction was made for contribution from particulate matter to the light-scattering measurements (see also Berth, 1988). The persistent presence of these 'microgel' contaminants (no matter how rigorous the clarification procedure) has been reported by other workers on pectins (see, e.g., Jordan \& Brant, 1978; Hourdet \& Muller, 1987). One method of reducing this problem is to couple GPC with low-angle laser light scattering and this has recently been applied to pectins by Brigand et al. (1990).

In this study we apply a somewhat different approach by using lowspeed sedimentation equilibrium measurements performed in an analytical ultracentrifuge to calibrate the GPC columns. Results from this technique are not generally coloured by the presence of these supramolecular particles - they are lost from optical registration at the cell base at the equilibrium speeds used.

We also use a related approach (sedimentation velocity) on the fractions - an approach which, when combined with the fraction molecular weights and/or intrinsic viscosity data, can be used to investigate the gross conformation (i.e. rod, coil or sphere) of these macromolecules in solution, from: (1) the Wales-Van Holde parameter viz., ratio of the concentration dependence sedimentation regression coefficient, $k_{s}$, to the intrinsic viscosity $[\eta]$ (Wales \& Van Holde, 1954; Cheng \& Schachman, 1955; Creeth \& Knight, 1965); (2) Mark-Houwink-Kuhn-Sakurada (MHKS) double-logarithmic representations of sedimentation coeffi- 
cient or intrinsic viscosity versus molecular weight (see, e.g. Harding et al., 1991); (3) rigid rod models; and (4) worm-like-coil models (Yamakawa \& Fujii, 1973, 1974; Garcia Molina et al., 1990).

\section{MATERIALS AND METHODS}

\section{Solvents}

The eluent for fractionation using GPC was a $0.037 \mathrm{M}$ phosphate buffer, $\mathrm{pH}=6.5$ with $1 \mathrm{~mm} \mathrm{Na}_{2}$ EDTA and $0.002 \% \mathrm{NaN}_{3}$. For sedimentation velocity and sedimentation equilibrium, a phosphate-chloride buffer was used, $\mathrm{pH}=6 \cdot 5, \mathrm{I}=0 \cdot 30$. The relevant proportions of $\mathrm{Na}_{2} \mathrm{HPO}_{4}$ and $\mathrm{KH}_{2} \mathrm{PO}_{4}$ were made up to a combined ionic strength of $0 \cdot 05$, and the higher ionic strength of 0.30 was attained by adding the relevant proportion of $\mathrm{NaCl}$ according to Green (1933). The pectins were dissolved at room temperature, and for sedimentation equilibrium extensively dialysed against the buffer. For sedimentation equilibrium, the solvent had $1 \mathrm{~mm} \mathrm{Na}_{2}$ EDTA+ $2 \mathrm{~mm} \mathrm{NaN}$ added.

\section{Pectin}

Citrus pectin (Koch-Light, UK) with a degree of esterification of $70 \%$ (methanol was determined by GPC after saponification) and a galacturonan content of $\sim 70 \%$ (obtained colorimetrically) (Blumenkrantz \& Asboe-Hansen, 1973) was used as the parent sample as before (Berth et al., 1990). Concentrations of pectin solutions for partial specific volume and sedimentation coefficient determinations were determined accurately by weight and corrected for moisture content.

\section{Gel Permeation Chromatography}

GPC was performed on Sepharose $2 \mathrm{~B} /$ Sepharose $4 \mathrm{~B}$ medium (total volume of $\sim 350 \mathrm{ml}$ ) with the $0.037 \mathrm{M}$ phosphate buffer. Solution $(15 \mathrm{ml})$ containing $30 \mathrm{mg}$ pectin was used for each GPC run without further purification. The pectin concentration in the eluate was monitored continuously using a differential refractometer (Knauer Instruments, FRG).

In order to produce sufficient material for subsequent sedimentation equilibrium and sedimentation velocity studies, pairs of neighbouring fractions were combined. Identical $20-\mathrm{ml}$ fractions from several GPC runs were collected, lyophilised, desalted on an Epidex B-2 column 
(MLW instruments, FRG) and lyophilised again. The carbohydrate content was calculated from the recovery at rechromatography.

\section{Partial specific volume determination}

The partial specific volume, $\bar{v}$, was determined by precision densimetry using an oscillating mechanical density meter (Anton-Paar Instruments, Austria) of the Kratky type (Kratky et al., 1973), thermostatted to $24 \cdot 8^{\circ} \mathrm{C} \pm 0 \cdot 1^{\circ} \mathrm{C}$. The instrument was calibrated using known concentrations of $\mathrm{CsCl}$ (after drying over $\mathrm{P}_{2} \mathrm{O}_{5}$ ) as standards (Crossley et al., 1982).

A linear plot of solution density versus concentration was obtained using the density meter (Fig. 1). Values for the density increment and partial specific volume of $0.434 \pm 0.010$ and $0.57 \pm 0.01 \mathrm{ml} \mathrm{g}^{-1}$, respectively were obtained.

\section{Sedimentation Equilibrium}

A Beckman (Palo Alto, USA) Model E analytical ultracentrifuge was used employing Rayleigh interference optics, an $\mathrm{He}-\mathrm{He}$ laser light source $(5 \mathrm{~mW}$, wavelength, $\lambda=632 \cdot 18 \mathrm{~nm}$ ) and an RTIC temperature measurement system. The intermediate speed method was used (Creeth \& Harding, 1982). In this method the speed is sufficiently low to avoid loss of resolution of the fringes near the base of the cell, so that highmolecular-weight components in the solution are not lost from optical registration. Because of the low speed, the meniscus concentration at

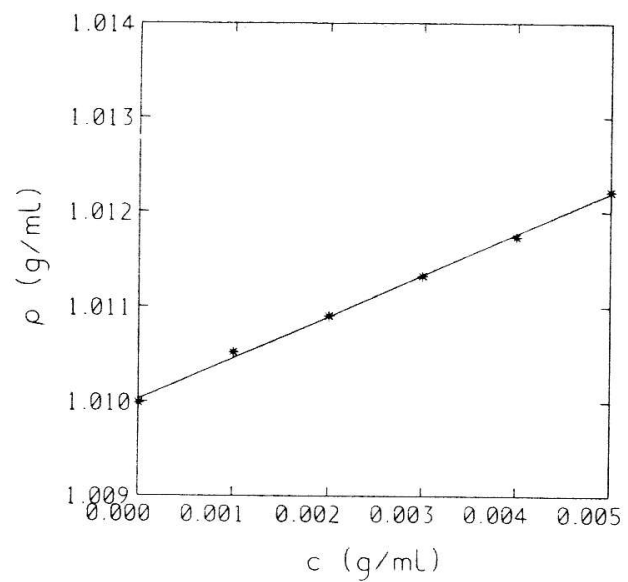

Fig. 1. Plot of density of unfractionated citrus pectin solutions vs. concentration. Buffer: phosphate/chloride, $\mathrm{pH}=6 \cdot 5, \mathrm{I}=0 \cdot 30$. Temperature $=24 \cdot 80^{\circ} \mathrm{C} \pm 0 \cdot 10^{\circ} \mathrm{C}$. 
equilibrium remains finite, and is obtained by mathematical manipulation of the fringe data (Creeth \& Harding, 1982). The (apparent) weightaverage molecular weight was determined from the limiting value at the cell base of a particularly useful operational point average (the 'star' average (Creeth \& Harding, 1982)). To minimise effects of thermodynamic non-ideality, all determinations were made at a low-loading concentration (approximately $0.7 \mathrm{mg} \mathrm{ml}^{-1}$ ) using $\sim 3 \mathrm{~mm}$ columns in double-sector cells of $30-\mathrm{mm}$ optical path length. The runs on fractionated material were performed in groups of three in multichannel 30-mm 'Yphantis-style' cells (Yphantis, 1960, 1964). An example of the Rayleigh interference patterns from these runs is given in Fig. 2.

\section{Sedimentation velocity}

Sedimentation velocity experiments were performed in an MSE (Crawley, UK) Centriscan Ultracentrifuge employing scanning schlieren optics with the monochromator set at $546 \mathrm{~nm}$. All experiments were performed at $20^{\circ} \mathrm{C}$ at rotor speeds of 40000-52000 rev min ${ }^{-1}$. Standard MSE $10-\mathrm{mm}$ and $20-\mathrm{mm}$ optical path length cells were employed. The apparent sedimentation coefficients $s_{\mathrm{s}(20, w)}$ at given concentrations, $c$ (milligrams per millilitre), were evaluated from the rate of movement of the Schlieren boundary using a computer digitising tablet to capture the data off-line. All concentrations were corrected for radial dilution effects.

Sedimentation coefficients were corrected to standard conditions (water as solvent at $20^{\circ} \mathrm{C}$ ) by using the usual formula (see, e.g., Tanford, 1961):

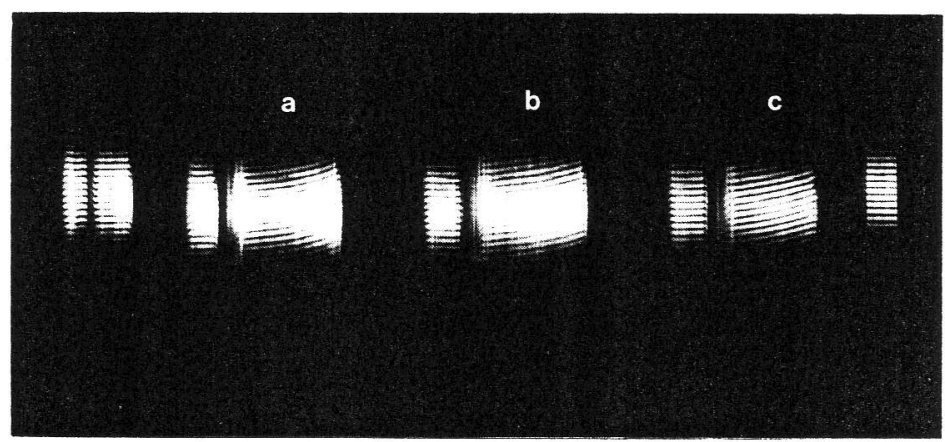

Fig. 2. Rayleigh Interference patterns from a low-speed sedimentation equilibrium experiment on citrus pectin fractions. A multi-channel 'Yphantis-style' $30-\mathrm{mm}$ path length cell was used. (a) fraction 15-16; (b) fraction 17-18; (c) fraction 19-20. The rotor speed was $9942 \mathrm{rev} \mathrm{min}^{-1}$, the temperature was $20^{\circ} \mathrm{C}$ and the initial loading concentration was $\sim 0.7 \mathrm{mg} \mathrm{ml}^{-1}$ in each case. Light source: $5 \mathrm{~mW} \mathrm{He}-\mathrm{Ne}, \lambda=632.18 \mathrm{~nm}$. 


$$
s_{\mathrm{c}(20, \mathrm{w})}=\frac{\left(1-\bar{v} \rho_{20, \mathrm{w}}\right)}{\left(1-\bar{v} \rho_{\mathrm{T}, \mathrm{b}}\right)} \frac{\eta_{\mathrm{T}, \mathrm{b}}}{\eta_{20, \mathrm{w}}} s_{\mathrm{c}(\mathrm{T}, \mathrm{b})}
$$

Here $\bar{v}$ is the partial specific volume, $\rho_{\mathrm{T}, \mathrm{b}}$ refers to the solution density and $\eta_{\mathrm{T}, \mathrm{b}}$ refers to the solvent viscosity. [In all that follows the symbol $s_{\mathrm{c}}$ is used in place of $\left.s_{\mathrm{c}(20, \mathrm{w})}\right] . s_{\mathrm{c}}$ values were extrapolated to 'infinite dilution' against concentration (corrected for radial dilution in the ultracentrifuge cell), to determine the infinite dilution sedimentation coefficient $(s)$ using the expression:

$$
1 / s_{\mathrm{c}}=(1 / s)\left(1+k_{\mathrm{s}}^{\prime} \cdot c\right)
$$

where $k_{\mathrm{s}}^{\prime}$ is the concentration dependence sedimentation regression coefficient for $s_{\mathrm{c}}$ values corrected for solvent density. This is further corrected for solution density (see e.g. Harding \& Johnson, 1985) using:

$$
k_{\mathrm{s}}=k_{\mathrm{s}}^{\prime}-\bar{v}
$$

Both $s$ and $k_{\mathrm{s}}$ were subsequently used for probing the gross conformation of the pectins.

\section{RESULTS AND DISCUSSION}

\section{Molecular Weight Distribution}

The elution profile from GPC is given in Fig. 3(a). The corresponding molecular weight distribution was evaluated following the procedure of Ball et al. (1988), as follows. An approximately linear Andrews-type plot of elution volume, $V_{\mathrm{e}}$ versus $\log M_{\mathrm{r}}$, the apparent weight average, from low-speed sedimentation equilibrium (Andrews, 1965) was obtained for the range analysed-Fig. 3(b). From the parameters for the line fitted (obtained by linear least-squares) the molecular weight distribution shown in Fig. 3(c) was obtained. The mode molecular weight was $\sim 60000 \pm 10000$ and the expected weight average of $\sim 97000$ calculated from this plot is in good agreement with the value of $90000 \pm 10000$ obtained from a separate low-speed sedimentation equilibrium experiment on unfractionated material.

The calibration line of Fig. 3(b) corresponds closely to that obtained using light scattering (after correction for particulate matter) described earlier (Berth et al., 1990). Within the central range of pectin elution $(160-260 \mathrm{ml})$ both calibration plots coincide almost exactly. Some small deviations are observed at the upper- and lower-molecular-weight limits 

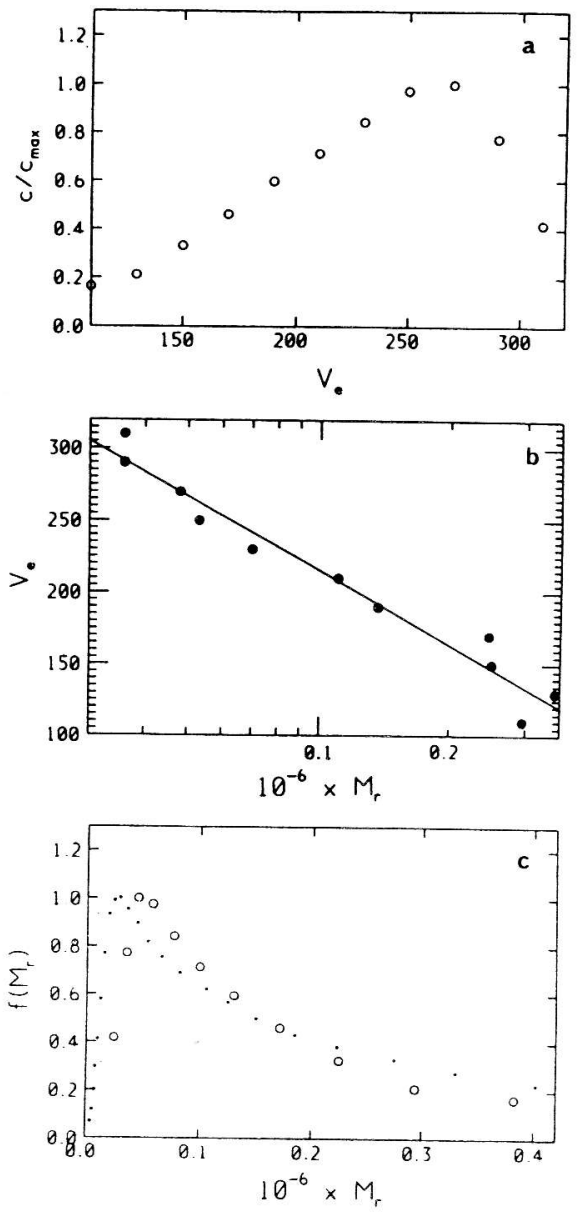

Fig. 3. Calibrated gel-permeation chromatography for Koch-Light citrus pectin. (a) Elution profile from a Sepharose $2 \mathrm{~B} /$ Sepharose $4 \mathrm{~B}$ column $\left(V_{0}=100 \mathrm{ml} ; V_{\mathrm{t}}=350 \mathrm{ml}\right)$. Loading concentration $\sim 2 \mathrm{mg} \mathrm{ml}^{-1}$, phosphate buffer, $\mathrm{I}=0 \cdot 15, \mathrm{pH}=6 \cdot 5$, flow rate $=11$ $\mathrm{ml} \mathrm{h}^{-1}$. (b) Calibration plot, using low-speed sedimentation equilibrium on isolated fractions of $\Delta V_{\mathrm{e}} \sim 20 \mathrm{ml}$. Points fitted to the line are described by $V_{\mathrm{e}}=a-K_{\mathrm{c}} \log _{10} M_{\mathrm{r}}$, with $a=1070.0 \mathrm{ml}$ and $K_{\mathrm{c}}=172 \mathrm{ml}$. (c) Corresponding molecular weight distribution (O). Also shown is the distribution of Berth et al. (1990), calculated on the basis of calibration using light scattering $(\bullet)$.

resulting in slightly different slopes of the calibration lines and hence a small shift of the molecular weight distribution curve of Fig. 3(c) compared with the observed distribution from light scattering/GPC - also shown in Fig. 3(c) (from Fig. 8 of Berth et al., 1990). Our calculated distribution would also appear to support a previous calibration using membrane osmometry (Berth et al., 1990). 


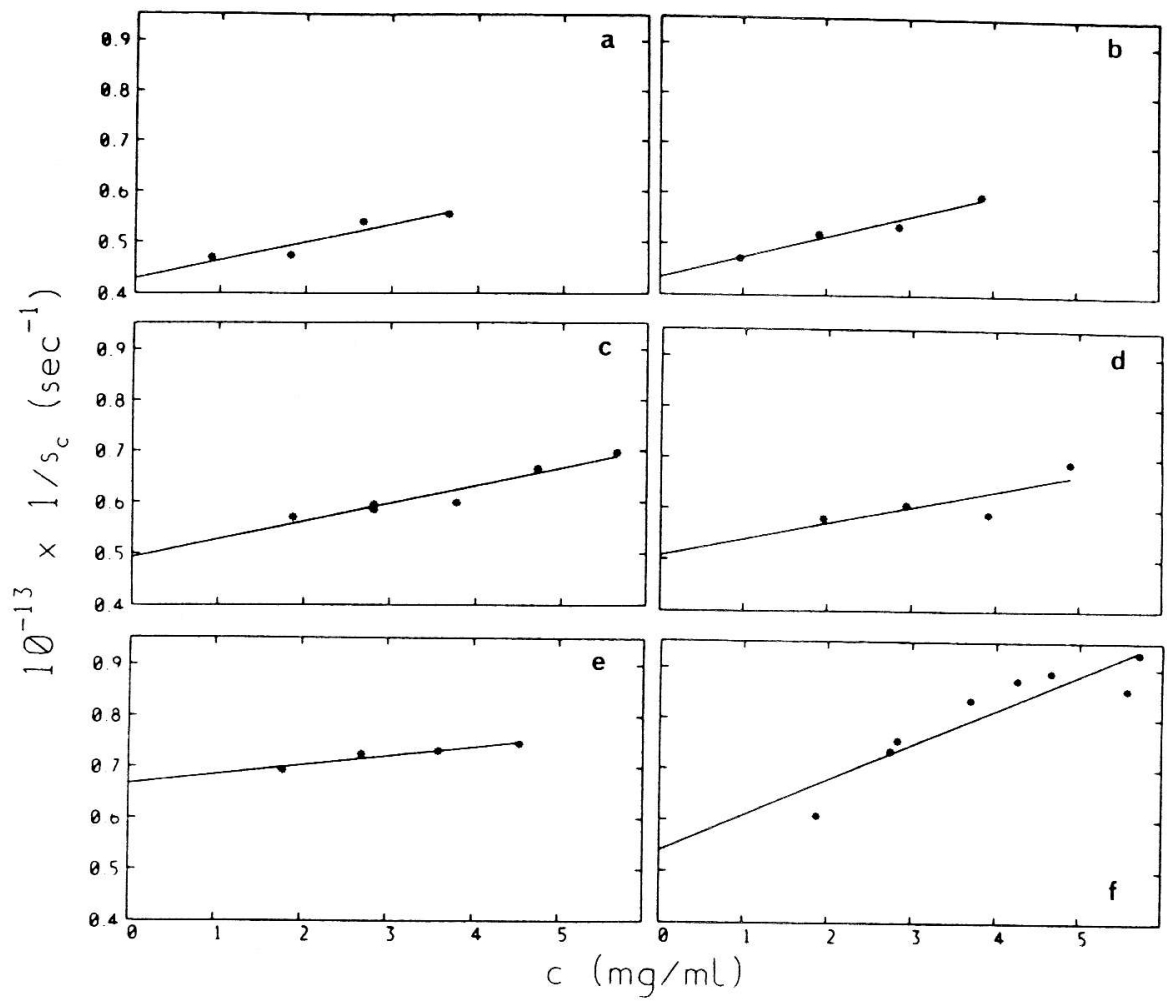

Fig. 5. Plots of the reciprocal of the sedimentation coefficient, $s_{c}$, vs. concentration for pectin fractions in phosphate $/ \mathrm{NaCl}$ buffer, $\mathrm{pH}=6.5$ and $\mathrm{I}=0.30$. All concentrations corrected for radial dilution effects. (a) fractions 9-10; (b) fractions 13-14; (c) fractions 15-16; (d) fractions 17-18; (e) fractions 19-20; (f) unfractionated.

been calculated from the weighted average of each set of two $10-\mathrm{ml}$ values and these values are also included in Table 1.

\section{Wales-Van Holde parameter, $k_{\mathrm{s}} /[\eta]$}

A useful indication of the possible conformation of the pectins in solution can be obtained from the Wales-Van Holde parameter (Wales \& Van Holde, 1954): the ratio of the sedimentation concentration dependence regression coefficient, $k_{\mathrm{s}}$, to the intrinsic viscosity, $[\eta]$. Whereas large values of $k_{\mathrm{s}}$ or $[\eta]$ could be due to high solvation or high asymmetry, or a contribution from both, the ratio $k_{\mathrm{s}} /[\eta]$ is a function only of asymmetry. The value of this ratio is characteristically $\sim 1.6$ for spheres and random coils, and substantially lower for asymmetric molecules (Wales \& Van Holde, 1954; Cheng \& Schachman, 1955; Creeth \& Knight, 1965; Harding \& Johnson, 1985). The values obtained for $k_{\mathrm{s}} /[\eta]$ are con- 

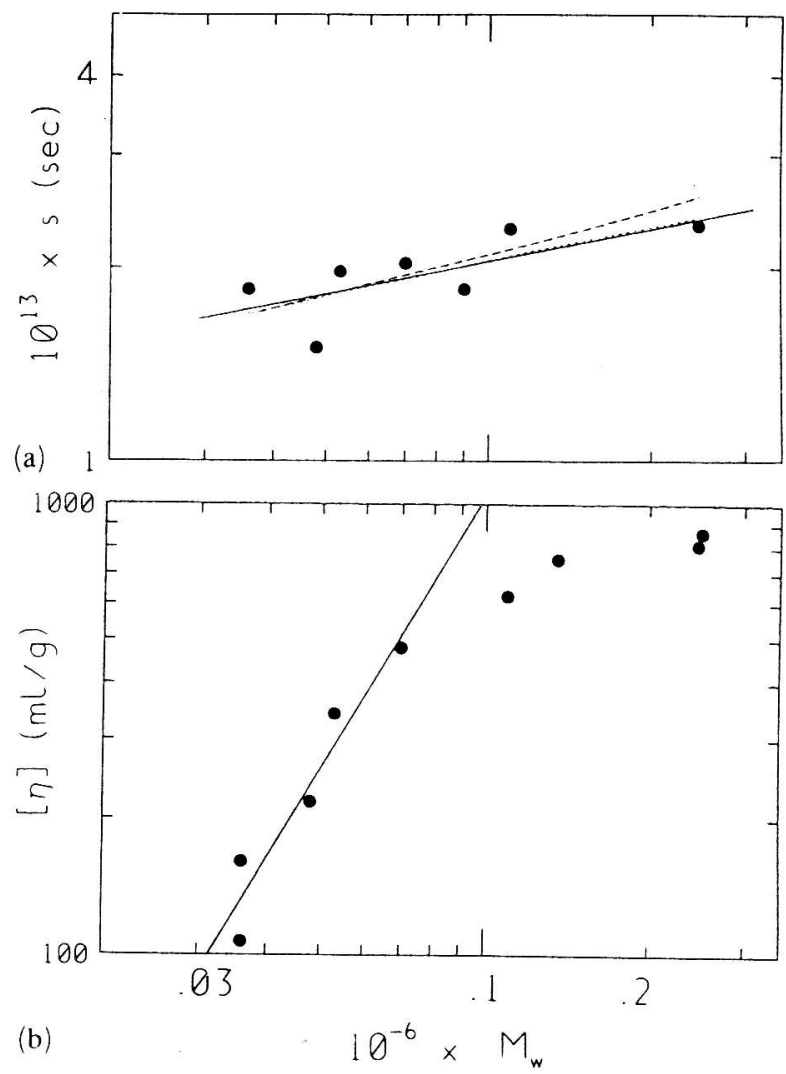

Fig. 6. (a) Double-logarithmic plot of sedimentation coefficient, $s$, vs. weight-average molecular weight, $M_{\mathrm{w}}$ (from sedimentation equilibrium) for citrus pectin fractions. Solid line fitted corresponds to the fit to the MHKS eqn - eqn. (4); slope $=(0 \cdot 17 \pm 0 \cdot 07)$. Dotted line (almost coincidental with the solid line) corresponds to the fit for a rigid rod model with $M_{\mathrm{L}}=430 \mathrm{~g} \mathrm{~mol}^{-1} \mathrm{~nm}^{-1}$ - eqn (6). Dashed line corresponds to the fit to a worm-like coil with large persistence length, $a$ (i.e. $\approx$ rod); $a=60 \mathrm{~nm}, M_{\mathrm{L}}=420 \mathrm{~g} \mathrm{~mol}^{-1}$ $\mathrm{nm}^{-1}$. (b) 'Double-log' plot of intrinsic viscosity vs. weight-average molecular weight, $M_{\mathrm{w}}$ (from sedimentation equilibrium). Solid line corresponds to a fit for the first five data points to the MHKS equation - eqn (5); slope $=2 \cdot 0 \pm 0 \cdot 3$ ).

A similar MHKS double-logarithmic plot was produced for the intrinsic viscosity, $[\eta]$, data plotted versus molecular weight - Fig. $6(\mathrm{~b})-$ to obtain the Mark-Houwink exponent $a$ in:

$$
[\eta]=K^{\prime} \cdot M^{\text {a }}
$$

Expected values for the extremes compact sphere, random coil and rigid rod are, respectively, 0, 0.5-0.8 and 1.8 (Smidsrød \& Andresen, 1979). The initial slope (estimated by linear regression of the first five data 
points) of the $\log [\eta]$ vs. $\log M_{\mathrm{w}}$ plot appears also to support the view that low-molecular-weight pectins behave as rods (slope $=2 \cdot 0 \pm 0 \cdot 3$ ). It is, however, significant to note that there is a decrease in slope with increase in molecular weight and there are two possible explanations here:

(1) Not shown in Fig. 6(b) are two points of higher molecular weight which have anomalously low values for the intrinsic viscosity. This is almost certainly due to the presence of appreciable amounts of compact high-molecular-weight spherical aggregates present in the solutions and noted earlier (Berth et al., 1990). If these species are also present (in proportionally lesser quantities) in the lower-molecular-weight fractions, this could provide an alternative explanation for the observed change in slope as molecular weight increases. Such effects would not be observed in the $\log s$ vs. $\log M_{\mathrm{w}}$ plot - Fig. 6(a) - since the sedimentation coefficients will not be affected (the high-molecular-weight material will be removed by the centrifugal field from optical registration).

(2) Another, perhaps more significant, contribution to the change in slope of Fig. 6(b) is a possible change in conformation of the pectin from a rod to a more coiled form as the molecular weight and the neutral sugar content increases. If data points are fitted over a wider range, the slope becomes more consistent with an extended coil shape conformation as noted before (Berth et al., 1990). The lack of evidence of this from the sedimentation velocity data could be a reflection of the view held by 'rigid-body' hydrodynamicists that the sedimentation coefficient is a less sensitive probe of shape than intrinsic viscosity.

\section{Rod model}

If we assume a rod model it is possible in principle to estimate the molecular parameters which describe the rod (Garcia Molina et al., 1990).

Starting with the sedimentation coefficient, the $s$ versus $\log M_{\mathrm{w}}$ data (and $\log s$ versus $\log M_{\mathrm{w}}$ data) is reasonably linear and a pure rod-like model can be assumed ( $c f$. the MHKS 'b' exponent above). The theoretical relation (Yamakawa \& Fujii, 1973) to be used for this model is:

$$
s=\frac{M_{\mathrm{L}}(1-\bar{v} \rho)}{3 \pi \eta_{0} N_{\mathrm{A}}}\left\{\ln M-\ln M_{\mathrm{L}}-\ln d+0.3863\right\}
$$

where $M_{\mathrm{L}}$ is the mass per unit length, and $d$ is the diameter of the rod. Recalling that $s$ is very insensitive to $d$, due to the logarithmic dependence, we take $d=0.7 \mathrm{~nm}$. Then eqn (6) allows the determination of the $M_{\mathrm{L}}$ from the experimental data, obtaining $M_{\mathrm{L}}=430 \mathrm{~g} \mathrm{~mol}^{-1}$ 
$\mathrm{nm}^{-1}$ - Fig. 6(a) - a value in good agreement with the expected value deducted from the mass and length of the pectate repeat unit.

The same approach, based on a purely rod-like model can be used for the first five $[\eta]$ data that seem to follow the slope of $\sim 2$ (the MHKS ' $a$ ' coefficient for rods). The pertinent equation is:

$$
[\eta]=\frac{Q \cdot N_{\mathrm{A}} \cdot M^{2}}{M_{\mathrm{L}}\left(\ln M-\ln M_{\mathrm{L}}-\ln d\right)}
$$

The most recent value for the coefficient $Q$ is 0.015 (Garcia Molina et al., 1990). We note that here $[\eta]$ is much more sensitive to the choice of $M_{\mathrm{L}}$. Unfortunately, no reasonable fit is obtained if an $M_{\mathrm{L}}$ of the expected order of magnitude (400-500 $\left.\mathrm{g} \mathrm{mol}^{-1} \mathrm{~nm}^{-1}\right)$ is used to fit the data of Fig. 6(b). A satisfactory fit to the first five data points of Fig. 6(b) can only be obtained for an $M_{\mathrm{L}} \sim 250 \mathrm{~g} \mathrm{~mol}^{-1} \mathrm{~nm}^{-1}$, which deviates appreciably from that obtained from the sedimentation velocity data, and is lower than the expected value from the pectate repeat unit: the viscosity data is too high by a factor of $\sim 2$ to yield an $M_{\mathrm{L}}$ of $\sim 430 \mathrm{~g} \mathrm{~mol}^{-1} \mathrm{~nm}^{-1}$. A good fit is obtained but only with an unreasonable value for $M_{\mathrm{L}}$ of $\sim 250 \mathrm{~g} \mathrm{~mol}^{-1} \mathrm{~nm}^{-1}$. It is unlikely that this phenomenon is due to the presence of microgels.

\section{Worm-like-coil-model}

A possibility of giving an improved fit to the viscosity and/or sedimentation data is use of a worm-like-coil model with the contour length, $L$, and the persistence length, $a$, as the key parameters. This gives a more comprehensive analysis of the properties and also caters for a rod model in the limit that $L \sim a$ (for a coil, $L>a$ ). Such a representation has been given before for pectins by Jordan and Brant (1978) (on the basis of representations of root mean square radius, $R_{\mathrm{g}}$, vs. $M_{\mathrm{w}}$ data) on the basis of $[\eta]$ vs. $M_{\mathrm{w}}$ data.

For flexible particles, curvature in plots of $\log s$ vs. $\log M_{\mathrm{w}}$ (upward curvature) and $\log [\eta]$ vs. $\log M_{\mathrm{w}}$ (downward curvature) would be expected, particularly for the latter which is generally more sensitive: such a flexibility effect can be properly described by the worm-like-coil model. Although the hydrodynamic properties of this model have been recently recalculated (Garcia Molina et al. 1990), the complexity of the newest theory and the experimental uncertainty in the experimental data (arising from e.g., sample polydispersity, etc.) would appear to justify the use of the simpler theory of Yamakawa \& Fujii $(1973,1974)$.

With the Yamakawa-Fujii $(1973,1974)$ theory and the simplified form of Bohdanecky (1983) for $[\eta]$, we carried out a least-squares simul- 
taneous fit with the persistence length $a$ and the mass per unit length $M_{\mathrm{L}}$ as the variables. From the sedimentation data, the best fit is achieved with $M_{\mathrm{L}}=420 \mathrm{~g} \mathrm{~mol}^{-1} \mathrm{~nm}^{-1}$ and $a=40-80 \mathrm{~nm}-$ Fig. 6(a). The value of $M_{\mathrm{L}}$ is practically the same as that found for the rod-like model (c), above, and the uncertainty in $a$ is due to the scatter of the data points and the small sensitivity of $s$ to flexibility. For a mass per unit length of this order, the sedimentation data is not consistent with anything other than a macromolecule which has rod-like characteristics. Although the curvature of the viscosity data - Fig. 6(b) - has the same form as would be expected for the worm-like coil, the actual data is significantly higher than would be expected for a mass per unit length of $400-500 \mathrm{~g} \mathrm{~mol}^{-1}$ $\mathrm{nm}^{-1}$. (A good fit is obtained only for an $M_{\mathrm{L}} \sim 230$ with a persistence length of $\sim 8 \mathrm{~nm}$ ).

We conclude that, on the basis of the sedimentation data, citrus pectins in the molecular weight range 20000-200000 have essentially rod-like characteristics in solution, with a mass per unit length of $\sim 430$ $\mathrm{g} \mathrm{mol}^{-1} \mathrm{~nm}^{-1}$. The low-molecular-weight intrinsic viscosity data is also consistent with a rod, although there are problems of interpretation arising from the possible presence of 'microgel' particles and values being too high to correspond to a meaningful mass per unit length.

\section{ACKNOWLEDGMENTS}

The expert technical help of Mr M. S. Ramzan is gratefully appreciated. This work was supported by UK AFRC Grant FG 42/218. The support of the joint UK-Spanish Acciones Integradas Program is gratefully appreciated.

\section{REFERENCES}

Andrews, P. (1965). Biochem. J., 96, 595.

Bailey, E., Mitchell, J. R. \& Blanshard, J. M. V. (1977). Colloid \& Polym Sci., 255, 856-60.

Ball, A., Harding, S. E. \& Mitchell, J. R. (1988). Int. J. Biol. Macromol., 10, 259. Berth, G. (1988). Carbohydr. Polym., 8, 105.

Berth, G., Dautzenberg, H., Lexow, D. \& Rother, G. (1990). Carbohydr. Polym., 12, 39 .

Blumenkrantz, N. \& Asboe-Hansen, G. (1973). Anal. Biochem., 54, 484.

Bohdanecky, M. (1983). Macromolecules, 16, 1483.

Brigand, G., Denis, A., Grall, M. \& Lecacheux, D. (1990). Carbohydr. Polym., 12,61 . 
Burton, B. A. \& Brant, D. A. (1969). Biopolymers, 22, 1769.

Cheng, P. Y. \& Schachman, H. K. (1955). J. Polym. Sci., 16, 19.

Creeth, J. M. \& Harding, S. E. (1982). J. Biochem. Biophys. Meth., 7, 25.

Creeth, J. M. \& Knight, C. G. (1965). Biochim. Biophys. Acta, 102, 549.

Crossley, J. M., Spragg, S. P., Creeth, J. M., Noble, N. \& Slack, J. (1982). Biopolymers, 21, 233.

Deckers, H. A., Olieman, C., Rombouts, F. M. \& Pilnik, W. (1986). Carbohydr. Polym., 6, 361.

Garcia Molina, M. C., Lopez Martinez, M. C. \& Garcia de la Torre, J. (1990). Biopolymers, 29, 883-900.

Green, A. A. (1933). J. Am. Chem. Soc., 55, 2331.

Harding, S. E. \& Johnson, P. (1985). Biochem. J., $231,543$.

Harding, S. E., Vårum, K., Stokke, B. T. \& Smidsrød, O. (1991). In Advances in Carbohydrate Analysis, Vol. 1, ed. C. White. JAI Press, Connecticut, in press.

Hourdet, D. \& Muller, G. (1987). Carbohydr. Polym., 7, 301.

Jordan, R. C. \& Brant, D. A. (1978). Biopolymers, 17, 2885.

Kim, W. J., Rao, V. N. M. \& Smit, C. J. B. (1978). J. Food Sci., 43, 572.

Kratky, O., Leopold, H. \& Stabinger, H. (1973). Meth. Enzymol., 27, 98.

May, C. D. (1990). Carbohydr. Polym., 12, 79.

Sheehan, J. K. \& Carlstedt, I. (1984). Biochem. J., $217,93$.

Smidsrød, O. \& Andresen, I. L. (1979). Biopolymerkjemi, Tapir, Trondheim, pp. 159, 255.

Tanford, C. (1961). Physical Chemistry of Macromolecules, Wiley, New York, Chap. 6.

Wales, M. \& Van Holde, K. E. (1954). J. Polym. Sci., $14,81$.

Yamakawa, H. \& Fujii, M. (1973). Macromolecules, 6, 407-15.

Yamakawa, H. \& Fujii, M. (1974). Macromolecules, 7, 128-35.

Yamakawa, H. \& Yoshizaki, T. (1980). Macromolecules, 13, 633-43.

Yphantis, D. A. (1960). Ann. N. Y. Acad. Sci., 88, 586.

Yphantis, D. A. (1964). Biochemistry, 3, 297. 